\title{
Suppression of MiR130a-3p Using CRISPR/Cas9 Induces Proliferation and Migration of Non-Small Cell Lung Cancer Cell Line
}

\author{
Nur Ainina Abdollah ${ }^{1}$, Nabil Izzatie Mohamad Safiai ${ }^{1}$, Muhammad Khairi Ahmad ${ }^{1}$, \\ Kumitaa Theva Das², Siti Razila Abdul Razak ${ }^{1, *}$ \\ ${ }^{1}$ Oncological and Radiological Sciences Cluster, Advanced Medical and Dental Institute, Kepala Batas, Pulau Pinang, 13200, Malaysia \\ ${ }^{2}$ Infectomics Cluster, Advanced Medical and Dental Institute, Kepala Batas, Pulau Pinang, 13200, Malaysia \\ *Corresponding author. E-mail: sitirazila@usm.my
}

Received date: Jul 27, 2021; Revised date: Sep 27, 2021; Accepted date: Sep 29, 2021

\section{Abstract}

ACKGROUND: Molecular alterations of microRNA130a (miR130a) are observed in many types of cancers, including non-small cell lung cancer (NSCLC). However, the role of miR130a in NSCLC has been poorly studied.

METHODS: In this study, clustered regularly interspaced short palindromic repeat (CRISPR)-Cas9 was utilised to knockdown miR130a. The gRNA was designed to target the stem loop, 3' and 5' sites of miR130a and stably expressed in A549 cells. Post-treatment, mature levels of miR130a$3 p$ and $5 p$ were quantified, and proliferation and migration assays were conducted.

\section{Introduction}

Lung cancer is listed as the most diagnosed cancer with $11.6 \%$ of the total cases, and the leading cause of cancer death with $18.4 \%$ of the total cancer deaths.(1) Among the different types of lung cancer, $80 \%$ was attributed by nonsmall cell lung cancer (NSCLC) with its major histological types, squamous cell lung cancer and adenocarcinoma.(2) Currently, many modalities of lung cancer treatment have emerged, from conventional therapies such as surgery, chemotherapy and radiotherapy, to targeted therapies and personalised medicine. Surgical resection is commonly performed for operable tumours of NSCLC, while chemotherapy and radiotherapy are the primary treatment
RESULTS: Result showed significant suppression of miR130a-3p and $-5 p$ by two and three-fold respectively, when the CRISPR/Cas9 targeted at the 3' site and stem loop of the miR130a gene. Suppression of miR130a-3p significantly increased the growth and migration of A549 cells, but no significant changes were observed in cells with suppressed expression of miR130a-5p.

CONCLUSION: Our encouraging results highlight that the suppression of the miR130a is achievable using CRISPR/ Cas9, and suppression of the miR-130a-3p could play an important role in the regulation of NSCLC.

KEYWORDS: miR130a, CRISPR-Cas9, non-small cell lung cancer

Indones Biomed J. 2021; 13(4): 364-74 of non-resectable tumours of NSCLC. However, there are many intolerable, harmful side effects with chemotherapy and radiotherapy. Other major hindrances to effective treatment include metastatic and relapse of the cancer.(3)

Thus, targeted therapy for specific molecular genomics alteration may be an alternative treatment option for patients. With an aim of to increase the survival rate of patients, an effective targeted agent in conjunction with chemotherapy and radiotherapy for locally advanced NSCLC patient is needed.(4) In addition, the personalised molecular targeted therapy is important to increase specificity of treatment as well as to increase the survival rate of the advanced stage lung cancer patients. One of the examples of molecular therapy that have been develop is targeted against two genetic abnormalities namely epidermal growth factor 
receptor (EGFR) mutation and anaplastic lymphoma kinase (ALK) gene rearrangements.(4) In accordance with that, microRNAs (miRNAs) which are $\sim 22$ nucleotides short stranded noncoding RNA, has been extensively studies and reported to play roles in various human biological activities including regulation of cell proliferation, apoptosis, cellular differentiation and immune responses.(5) As they are an important component of cell regulation, miRNAs have been used for cancer treatment, including miRNAs replacement therapy, miRNA inhibition therapy and miRNA combination therapy.(6,7) An miRNA replacement therapy, MRX34, is a liposome-based miR34 mimic which is in clinical trial phase I for treatment of hepatocellular carcinoma (HCC). (6) Another study showed that co-delivery of docetaxel with miR34a for treatment of metastatic breast cancer provided a synergystic therapeutic effect (7), highlighting the significance of miRNA therapy in cancer.

MiR130a has been studied numerous times and could either promote or inhibit cancer growth in different types of tumours depending on its level of expression. It is indicated to be involved in cancer development, progression, and metastasis as it was reported to be upregulated in several types of cancer including colon cancer, chronic myeloid leukaemia and hepatocellular carcinoma.(8-10) In prostate cancer, miR130a is expressed at low levels, and restoration of the level of miR130a to normal levels reduced the malignancy of cancer.(11) In addition to that, miR130a is expressed at low levels in breast cancer patients, and rescuing the downregulated miR130a inhibited cellular proliferation, invasion and migration of invasive breast cancer.(12,13)

In addition, several studies have shown that miR130a was found to be down-regulated in NSCLC and in various chemoresistant tumour cells.(14-16) In lung cancer, miR130a was reported to control the expression of miR221/222 and affected the cell migration and invasion of the lung cancer cell line.(17) Recently, miR130a was reported to be involved in the regulation of lung cancer growth through Kruppel Like Factor 3.(18) Although several studies demonstrated the important role of miR130a in various cancers, there is scarce knowledge regarding the function of miR130a in lung cancer. Hence, in this study, the molecular activity of the miR130a and its regulatory role in lung cancer is investigated using DNA loci with short repetitions of base sequences known as CRISPR. Using this system, the Cas9 which is a nuclease guided by small RNAs will introduces double stranded break (DSB) at the targeted genomic locus. (19) The DSB is then repaired through non-homologous end joining, which introduces random insertions and deletions at the break site, causing frame shifts, and consequently, the disruption of the gene function. It was reported in different cell types and organisms that the CRISPR-Cas9 is highly specific, efficient and well-suited for high throughput and multiple gene editing. $(19,20)$ Hence, in this study, CRISPRCas9 system is used to suppress the endogenous expression of mature miR130a in NSCLC cell line, with the aim of to investigate the effect of miR130a suppression on cell proliferation and migration.

\section{Methods}

\section{Cell Culture}

A549 (human bronchioloalveolar lung carcinoma) was obtained from American Type Culture Collection (ATCC) and cultured in RPMI 1640 (Gibco, USA) medium supplemented with $10 \%$ heat inactivated fetal bovine serum (FBS) and $1 \%$ antibiotics $(10,000 \mathrm{U} / \mathrm{mL}$ of penicillin, 10,000 $\mu \mathrm{g} / \mathrm{mL}$ of streptomycin) (Gibco, Waltham, MA, USA). Cells were grown in a $37^{\circ} \mathrm{C}$ incubator at $5 \% \mathrm{CO}_{2}$.

\section{Design of Single Guide RNA (sgRNA)}

For site-specific cleavage of pre-miR-130a target regions, the sgRNAs were designed using the CRISPR MIT tool and were cloned into lentiCRISPRv2 plasmid. The plasmid was a gift from Feng Zhang (Addgene plasmid \#52961; http:// n2t.net/addgene:52961; RRID: Addgene_52961).(21) The lentiCv2-miR130_3p was designed to target the $3 p$ region of mature sequence of miR130a, the lentiCv2-miR130 5p was designed to target the $5 \mathrm{p}$ region of mature sequence of miR130a while lentiCv2-miR130_SL was designed to target the stem-loop region of mature sequence of miR130a. Briefly, the $88 \mathrm{bp}$ of pre miR130a sequence was retrieved from National Centre for Biotechnology Information (NCBI) with reference sequence of NC_000011.10, and was used for sgRNAs design.(22) The sequence of 5'-TGC TGC TGG CCA GAG CTC TTT TCA CAT TGT GCT ACT GTC TGC ACC TGT CAC AGC AGT GCA ATG TTA AAA GGG CAT TGG CCG TGT AGT G-3' was submitted to the CRISPR MIT tool (http://crispr.mit.edu) for identification of sgRNAs. Overhang for BsmBI restriction site was included in the sense strand (oligo 1) and in the antisense strand (oligo 2) of the sgRNAs. The respective guide RNA sequences are as follows: gRNA targeted at the $3 p$ of miR130a are Oligo 1: CAC CGT GCA ATG TTA AAA GGG CAT, and Oligo 2: AAA CAT GCC CTT TTA ACA TTG CAC ; gRNA targeted at the 5p of miR130a are Oligo 1: CAC CGC ACA ATG TGA AAA GAG CTC and 
Oligo 2: AAA CGA GCT CTT TTC ACA TTG TGC while the gRNA targeted at the SL region are Oligo 1: CAC CGA ACA TTG CAC TGC TAG TGA and Oligo 2: AAA CGT CAC TAG CAG TGC AAT GTT. Oligos 1 and 2 of each target site was annealed and cloned into lentiCRISPRv2 plasmid. Selection of positive clones were carried out using PCR colony, and the selected clones were sent for sequence validation. The positive clones were propagated in LB broth (with $50 \mu \mathrm{g} / \mathrm{mL}$ ampicillin), and the plasmid DNA was extracted and purified using Endotoxin-free plasmid DNA purification, NucleoBond® Xtra Midi EF (Machery-Nagel, Germany) according to manufacturer's protocol.

\section{Plasmid Transfection and Selection of Successfully Transfected Cells}

$5 \mu \mathrm{g}$ of each cloned plasmid (lentiCv2-miR130a_3p, lentiCv2-miR130a_5p and lentiCv2-miR130a_SL) was transfected into A459 cells using Lipofectamine ${ }^{\circledR} 3000$ (Life Technologies, Waltham, MA, USA) according to manufacturer's protocol to produce A549-3p, A549-5p, and A549-SL. Empty lentiCv2_CT plasmid transfected to A549 cells to produce A549-CT and was used as a negative control. The transfected cells were maintained in a $37^{\circ} \mathrm{C}$ incubator, humidified at 5\% $\mathrm{CO}_{2}$. At 5-hours post-transfection, $1 \mathrm{~mL}$ of complete media was added to each well, and at 24-hours post-transfection, the medium in each well was replaced with fresh complete media. At 48-hours post-transfection, the medium in each well was replaced with complete media and $0.75 \mu \mathrm{g} / \mathrm{mL}$ puromycin (Merck, Darmstadt, Germany). Cells were maintained in the selection medium with puromycin for 72-hours. Cells that survived after 72-hours of culture were further maintained in puromycin-free complete growth medium, and the silencing activity was validated using qRT-PCR and other functional assays.

\section{Quantitative Real-time PCR (qRT-PCR)}

miRNA extraction including total RNA was conducted using miRNeasy Mini Kit (Qiagen, Germantown, MD, USA) according to manufacturer's protocol. RNA was extracted from $5 \times 10^{6}$ to $5 \times 10^{7}$ cells. TaqMan microRNA Reverse Transcription Kit (Applied Biosystems, Waltham, MA, USA) and TaqMan ${ }^{\circledR}$ Universal Master Mix II, no UNG (Applied Biosystems) were used in this twostep RT-PCR. Each sample was briefly centrifuged and transferred to a thermocycler, StepOnePlus ${ }^{\text {TM }}$ Real-Time PCR Systems (Thermo Fisher Scientific, Waltham, MA, USA). The qRT-PCR was used to measure the expression of mature miR130a-3p (hsa-miR-130a-3p, Thermo Fisher Scientific) and miR130a-5p (hsa-miR-130a-5p, Thermo
Fisher Scientific) to validate the silencing activity of the CRISPR-Cas9 system. The calculations were made based on comparative $\mathrm{CT}$ method $\left(2^{-\triangle \Delta C T}\right)$ expression. RNU6B (RNU6B, Thermo Fisher Scientific), a housekeeping gene was used as a positive control as it stably expresses snoRNAs in comparison to other human reference genes.(23)

\section{A549 Growth Proliferation Assay}

The A549-CT, A549-3p, A549-5p and A549-SL were seeded into 24 well plates at $2.5 \times 10^{4}$ cells per well and were maintained in complete RPMI 1640 media and harvested at 24-hour intervals until day 5. Harvested cells were trypsinized and live cells were counted under 10x objective (Axiovert $40 \mathrm{CFL}$ ). Data for cell growth at zero hour was normalized to $100 \%$. The experiments were performed in biological triplicates.

\section{Scratch Wound Healing Assay}

An optimization was performed to synchronize cell cycle and deplete growth factors that may influence cell migration, A549-CT cells were cultured overnight and after 24-hours, the media was replaced with new media supplemented with $1 \%$ FBS. Then, the number of A549-CT cells were calculated at 16, 20 and 24-hours. The A549-CT cells continued to proliferate when supplemented with $10 \%$ FBS. The assay was performed by culturing $2 \times 10^{5}$ A549 cells into 24-well plates. The cells were allowed to proliferate overnight to reach $97-100 \%$ confluency. Then the cells were incubated with RPMI media supplemented with only 1\% FBS. A 200 $\mu \mathrm{L}$ pipette tip was used to scratch the confluent monolayer. The well was washed thrice to remove floating cells. Then, the cells were incubated to allow migration into the acellular area. Cells were photographed at 0, 24, 48 and 72-hours using light microscope (Olympus CKX41, Olympus, Tokyo, Japan). Pictures of confluent monolayer were taken as prescratch images at $10 \mathrm{x}$ objective view prior to the initial scratch at 0-hour. All images were analysed by tracing the border of the wounded area using Image J software (Image $\mathrm{J} 1.52 \mathrm{~b}$ version). The results were displayed as a percentage of wound closure. The equation used is as follows: \% of wound closure $=\left[\left(\mathrm{A}^{\mathrm{t}} 0 \mathrm{~h}-\mathrm{A}^{\mathrm{t}} \Delta \mathrm{h}\right) / \mathrm{A}^{\mathrm{t}} 0 \mathrm{~h}\right] \times 100 . \mathrm{A}^{\mathrm{t}} 0$ $\mathrm{h}$ is the initial area of the gap measured immediately after scratching, and $\mathrm{A}^{\mathrm{t}} \Delta \mathrm{h}$ is the area of wound measured at either 24, 48 or 72-hours after scratching.(24)

\section{Attachment and Detachment Assay}

The attachment and detachment assays were performed as described previously.(25) In the attachment assay, $5 \times 10^{4}$ cells were plated onto 24-well plates and incubated for 
1-hour. After an hour, the unattached cells were discarded. The attached cells were incubated with $0.05 \%$ trypsin for approximately 10 minutes until cells were detached. Once detached, cells were counted under the inverted light microscope. Results were displayed as a percentage of the attached cells over total cells. Equation used for attachment assay is as follows: $\%$ of attached cells $=$ (number of cells counted after trypsinization/ number of cells seeded) x 100 .

For the detachment assay, cells were seeded and incubated for 24-hours. Following that, the media was discarded, and the cells were incubated with $0.05 \%$ trypsin for 3 minutes. Then, the trypsin activity was inactivated with the addition of culture media containing FBS. All detached cells were collected and counted. Subsequently, the remaining cells were incubated and detached using $0.25 \%$ trypsin. Once the activity of the trypsin was inactivated, all detached the cells were collected and counted. All experiments were performed using biological triplicates. The results were displayed as a percentage of detached cells over total cells following equation as follows: $\%$ of detached cells $=[$ number of cells detached by $0.05 \%$ trypsin/ number of all detached cells] x 100.

\section{Statistical Analysis}

Data was analysed using Student's t-test (one-tailed distribution, two-sample equal variance) and shown as mean \pm standard deviation (SD) of biological triplicates. $p$-value of significant differences were noted as $* p \leq 0.05$, $* * p \leq 0.01$.

\section{Results}

\section{Validation of miR130a Suppression in A549 Cell Line} CRISPR-Cas9 silencing activity of miR130a-3p and miR130a-5p were validated by quantifying the relative expression of mature miR130a-3p and miR130a-5p on day 4 and day 28 of puromycin selection. The expressions were compared relatively to A549-CT transfected with lentiC_miR130a-CT, for comparative CT method $\left(2^{-\Delta \Delta C T}\right)$ expression analysis. Relative quantification results showed significant down regulation of miR130a-3p by 2-fold $\left({ }^{*} p \leq 0.05\right)$ in A549-3p transfected with lentiC_miR130a-3p on day 4 of puromycin selection (Figure 1A). Significant down regulation of miR130a-5p by 3 -fold $\left({ }^{*} p \leq 0.05\right)$ was observed in A549-SL transfected with lentiC_miR130a-SL (Figure 1B). The expressions of mature miR130a-3p and $5 p$ were re-analysed on day 28 of puromycin selection to investigate the long-term suppression of miR130a-3p and
miR130a-5p. Relative quantification result showed similar patterns of suppression, where miR130a-3p expression was suppressed by 2 -fold in A549-3p $\left({ }^{*} p \leq 0.05\right)$ and 3 -fold $\left({ }^{*} p \leq 0.01\right.$ ) of miR130a-5p in A549-SL (Figure 1C, Figure 1D). No significant difference in the expression of miR130-3p and miR130-5p was observed in A549-5p. This result strongly proves significant long-term CRISPR/ Cas9 silencing activity of miR130a-3p and miR130a-5p expression in A549-3p and A549-SL, respectively.

\section{Effects of Suppression on the Cells Morphology}

A549-3p and A549-SL were selected for further investigation based on the suppression of miR130-3p and miR130-5p in those cell lines. The stably transfected cells were cultured at passage 17 and the morphology of the cells was observed at 24, 48 and 96 hours of culture under $10 \mathrm{x}$ objective (Figure 2A). Control A549-CT cells were observed to have elongated fibroblastic-like morphology while A549-3p cells were noted to have more elliptical shape with less cytoplasm compared to A549-CT and A549-SL. A549-3p cells also had very refined edges. There were no obvious differences in the histological shape between A549-SL and A549-CT.

\section{miR130a Can Suppress A549 Lung Cancer Cells Proliferation}

To investigate the function of miR130a-3p and miR130-5p on cell growth in vitro, the growth of A549-CT, A549-3p and A549-SL were monitored at 24-hour intervals for 5 consecutive days. Viable cells were counted using trypan blue and haemocytometer, and the percentage increment from 0-hour was calculated and tabulated in a bar graph as shown in Figure 2B. The results showed that there were no significant differences in growth percentage between A549$3 p$ cells and A549-SL cells compared to A549-CT cells at 24 and 48-hours of culture. At 72-hours of culture, A549$3 p$ and A549-SL were shown to have significant growth percentage ( $p \leq 0.01)$ with $631.7 \%$ and $662.1 \%$ respectively, when compared to A549-CT. At 96-hours, compared to A549-CT, both A549-3p and A549-SL were shown to have significant increases in the percentage of cell growth with $p \leq 0.05$ with $839.4 \%$ and $902 \%$, respectively. At 120 -hours, no significant difference in the percentage of cell growth was observed across all groups.

\section{Suppression of miR130a-3p Promoted Migration of Cells in Scratch Wound Healing Assay}

To further investigate whether miR130a-3p and miR130$5 p$ affects cell migration, scratch wound healing assays were performed. Figure 3 shows the results of scratch 
A

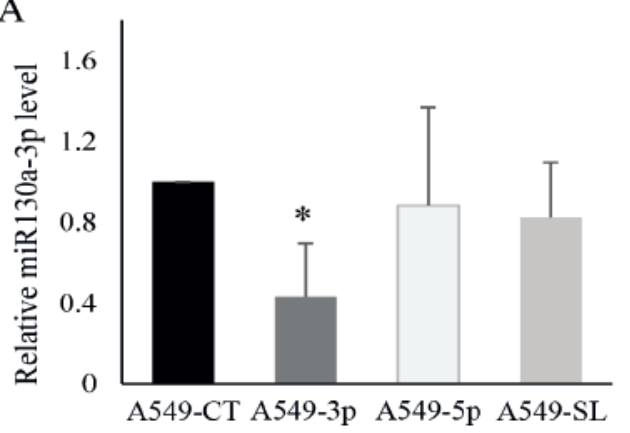

$\mathrm{C}$

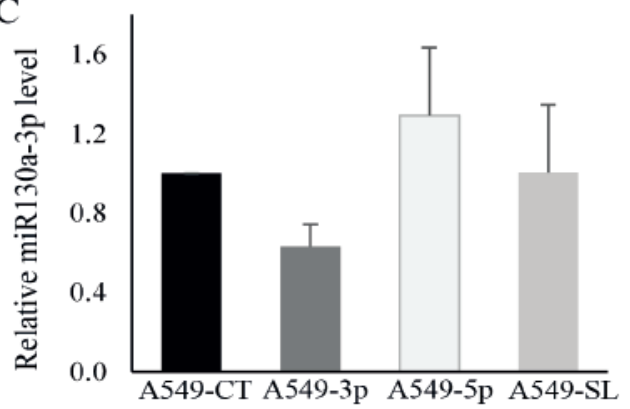

B

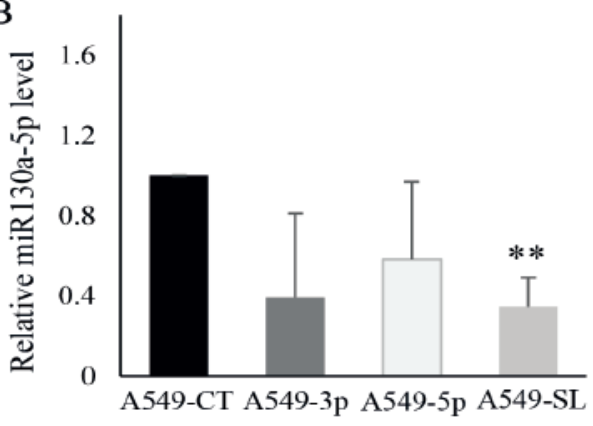

D

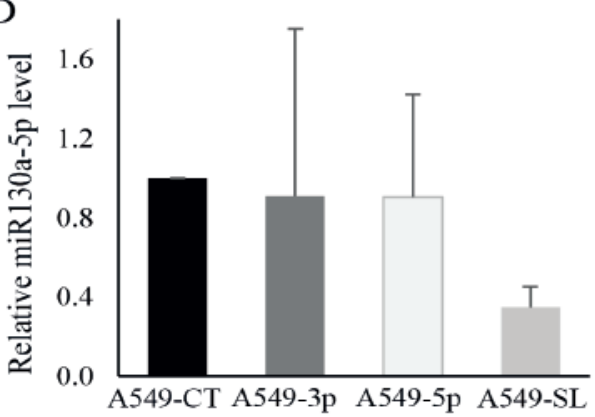

Figure 1. qRT-PCR was performed to measure the expression of mature miR130-3p and miR130a-5p. miR130-3p (A) and miR130$5 p$ (B) were measured in all transfected cells on day 4 of puromycin selection. On day 28 of selection, the relative expression of miR 130a$3 p(C)$ and miR130a-5p (D) were measured again. The relative expression was normalised to A549-CT. Values shown as mean $\pm \mathrm{SD}(\mathrm{n}=3$, ${ }^{*} p \leq 0.05,{ }^{* *} p \leq 0.01$ ) using Student's t-test (one-tailed distribution, two-sample equal variance).

wound healing assay on A549-CTs, A549-3p and A549SL. After 24-hours of culture, the cells were viewed under a light microscope, and A549-CT, A549-3p and A549-SL had migrated to close the wounded gap area as shown in Figure 3A. After 48-hours from the initial scratch, A549-3p had migrated more compared to A549-CT cells and A549SL cells. At 72-hours, A549-3p had almost completely closed the wounded gap area in each well. Based on the observations, A549-3p had the highest migration rate compared to A549-CT and A549- SL. Data from the scratch wound healing assay was analysed and percentages of wound gap closure were tabulated as displayed in Figure 3B. At 0 -hours, the wounded areas were measured, and all the gap closures were set to $0 \%$. All percentages increased at 24, 48, and 72-hours. There is an inverse correlation between the percentage of wound gap closure to gap of the wounded area, and a positive correlation between the percentage of wound gap to the migratory ability of the cells. So, the higher the percentage of wound gap closure, the smaller the gap of the wounded area and the higher the migratory ability of the cells. The A549-3p cells showed a significant percentage of wound closure with $33.18 \%$ at 24-hours $(p \leq 0.05), 56.29 \%$ at 48 -hours $(p \leq 0.05)$ and
$88.91 \%$ at 72 -hours $(p \leq 0.01)$. Meanwhile, A549-SL cells had a $21.95 \%$ of wound gap closure at 24 -hours, $28.15 \%$ at 48 -hours and $43.7 \%$ at 72 -hours. Overall, these results suggest that suppression of miR130a-3p significantly increases the numbers of cells migrating across the wound in A549-3p cells.

Suppression of miR130a-3p Promoted the Cell Attachment and Detachment

Attachment and detachment assays were done to determine the adhesive capability of A549 cells. As presented in Figure $3 \mathrm{C}$, in the attachment assay, A549-3p cells were shown to have a significantly higher adhesion with $32.7 \%$ cell attachment $(p \leq 0.05)$ compared to A549-CT cells with $25.7 \%$ of attached cells. A549-SL cells had a lower percentage of cell attachment at $19.7 \%$ compared with A549-CT cells The results of detachment assay are shown in Figure 3D. The A549-CT cells had 34.6\% of detached cells in the cell detachment assay. When compared to the control, all post-transfected A549 cells had a significantly higher percentage of detached cells. A549-3p cells had 56\% of detached cells $(p \leq 0.05)$ while A549-SL cells had $52.1 \%$ $(p \leq 0.05)$ of detached cells after trypsinisation. 
A

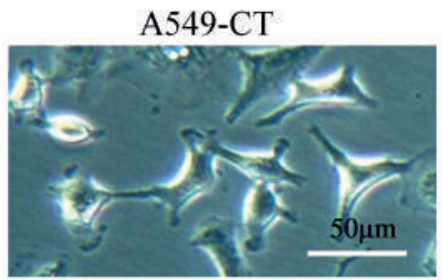

$24 \mathrm{~h}$

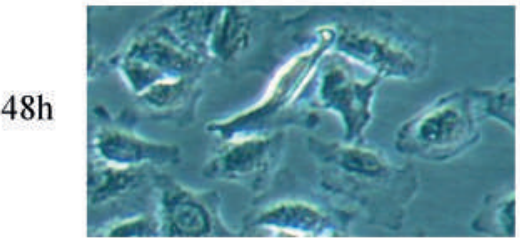

$96 \mathrm{~h}$

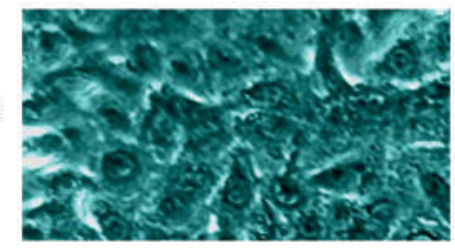

A549-3p
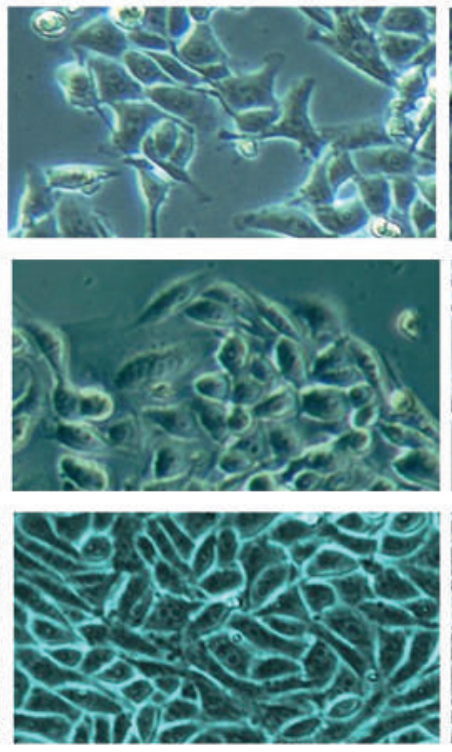

A549-SL
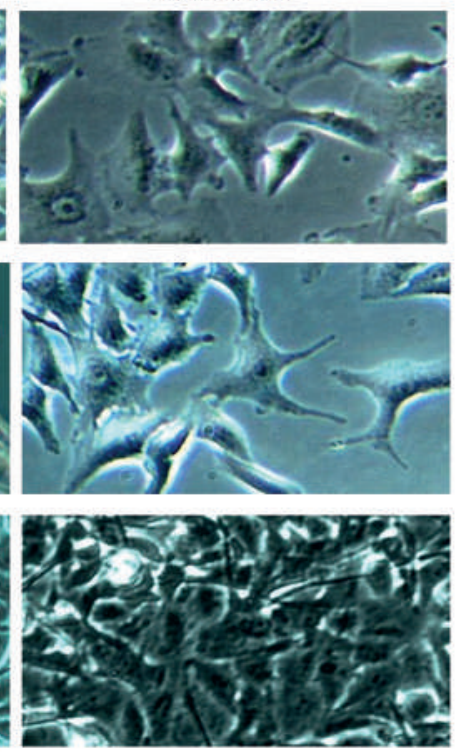

B

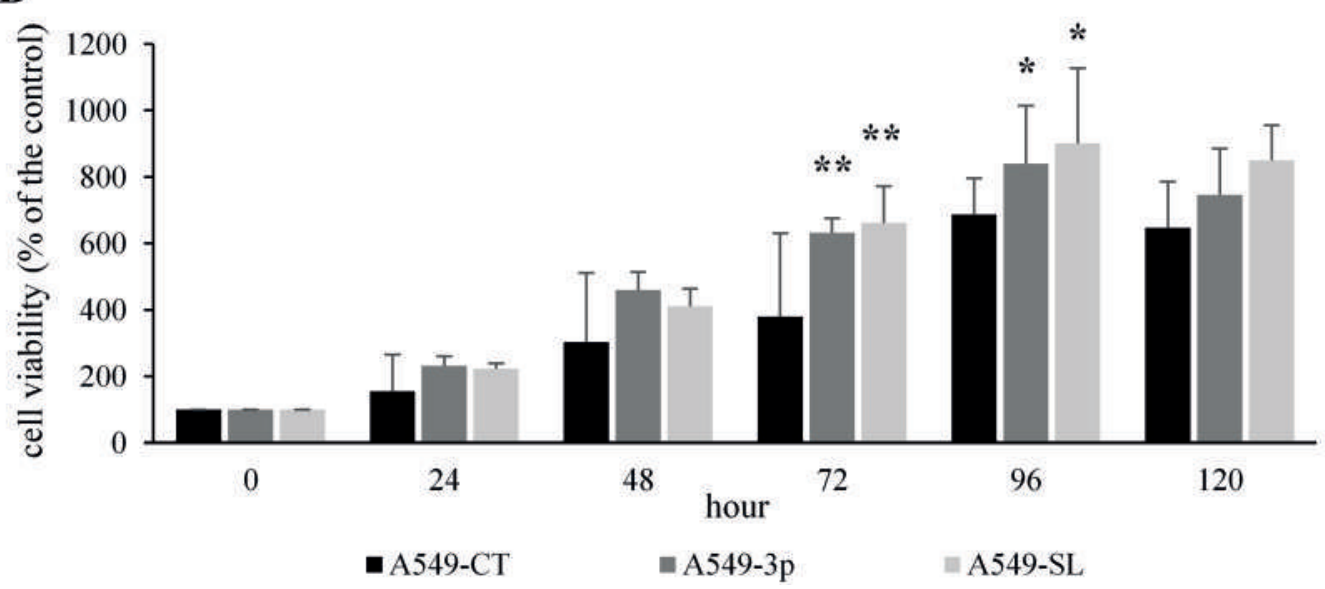

Figure 2. Effects of miR130a-3p and -5p suppression on cell morphology (A) and proliferation (B) of stably transfected A549 cells. The cells were viewed under $10 \mathrm{x}$ objective at 24,48 and 96 -hour respectively. Values shown as mean $\pm \mathrm{SD}\left(\mathrm{n}=3,{ }^{*} p \leq 0.05, * * p \leq 0.01, v s\right.$. control) using Student's t-test (one-tailed distribution, two-sample equal variance).

\section{Discussion}

In this study, we have shown that suppression of miR130a$3 p$ and $-5 p$ can be achieved in A459 on days 4 and 28 in an in vitro culture system using CRISPR-Cas9 system. We demonstrated that the lentiCv2-miR130_3p targeting at the $3 p$ region of miR130a gene is involved in the regulation of miR130a-3p expression. However, further investigations are needed to understand how $3 p$ region specifically regulates and controls the processing of miR130a-3p. qRT-
PCR analysis in A549-5p showed that there is no downregulation of miR130a-3p and $-5 p$ expression observed, suggesting that the $5 \mathrm{p}$ region of miR130a gene is unlikely to be involved in the regulation of miR130a-5p. This could be due to $-5 \mathrm{p}$ region playing a smaller role in the specificity of binding and cleavage of miR130a, as suggested by the minimal base pairing between the 'seed' sequence on $5 \mathrm{p}$ region with the targeted genomic DNA sequences.(26) On the other hand, downregulation of mature miR130a$5 p$ expression was observed in cells transfected with sgRNA targeted at the SL region of miR130a gene. The 

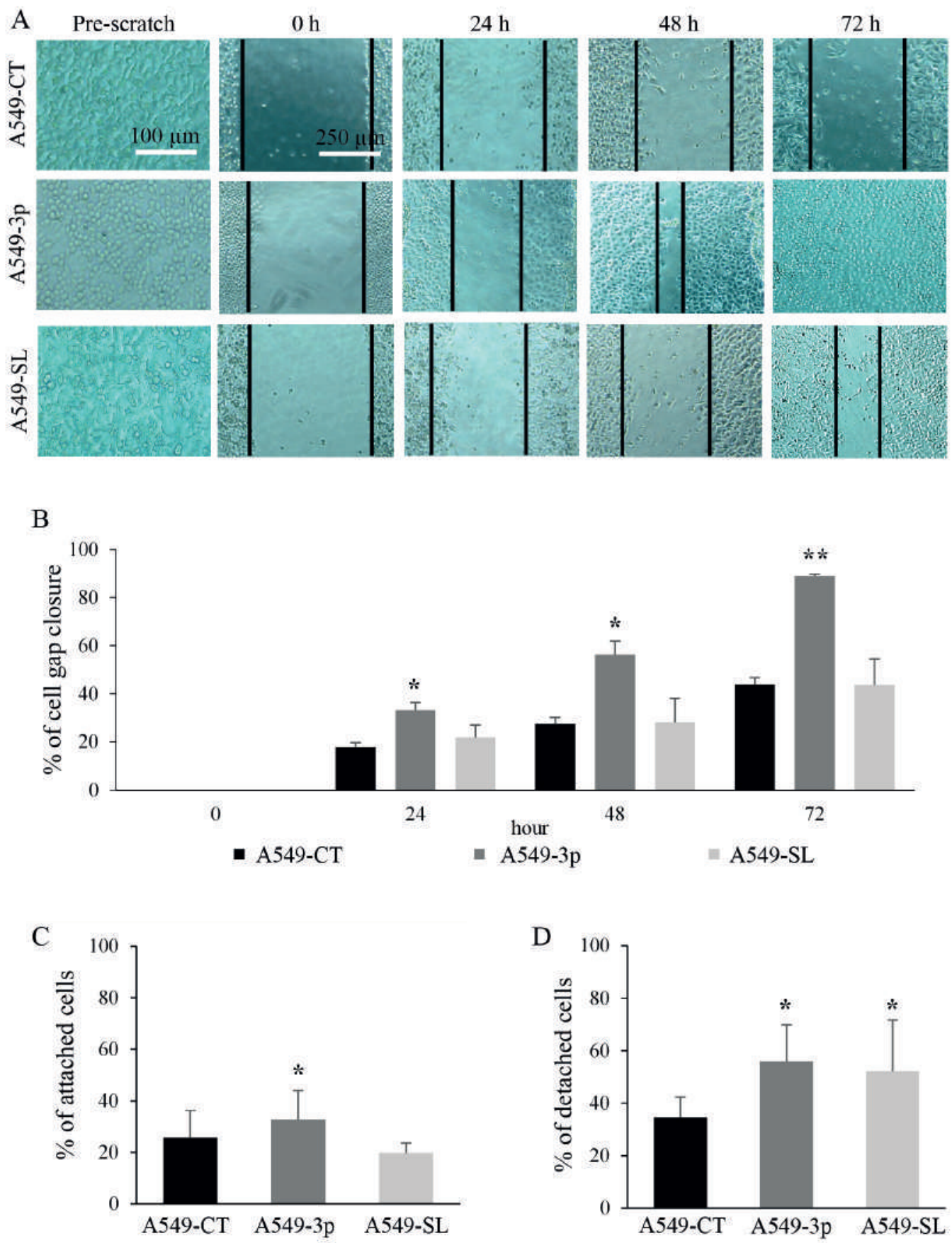

Figure 3. Down-regulation of miR130-3p and $\mathbf{- 5 p}$ in A549 cells. A: Scratch wound healing assay was performed to investigate the migratory potential of stably transfected A549 cell. B: The percentage of gap closure was calculated on the stably transfected A549 cell. C and D: Cells attachment and detachment assays were conducted after miR130-3p or miR130-5p suppression. Values shown as mean \pm SD ( $\mathrm{n}=3,{ }^{*} p \leq 0.05,{ }^{*} p \leq 0.01, v s$. control) using Student's t-test (one-tailed distribution, two-sample equal variance).

result suggests that the stem loop region probably plays a key role in controlling the regulation of mature miR130a$5 \mathrm{p}$. This is supported by previous studies which showed that SL binding proteins regulate the processing of priand pre-miRNA either by increasing or decreasing the miRNA biogenesis efficiency.(27-29) The processing of pri- and pre-miRNA of the let-7 family were inhibited by the interaction of Lin28 with the stem loop region.(27-29) However, another study had contradictory results as they showed that neither the sequence nor the loop structure is important in the recognition of pre-miRNAs by exportin-5. $(30,31)$ They stated that only the length of both $3 p$ and $5 p$ regions, and the presence of 39 nucleotide overhangs in the 3 ' strand of miRNA are important for the recognition of 
exportin-5.(30,31) Contradictory to that, our study showed that the stem loop region is involved in the regulation of miR130a-5p expression. However, downstream analyses need to be conducted to identify the mechanism of how stem loop of miR130a regulating the processing and thus expression of miR130a-5p.

MiR130a has been proven to be lowly expressed in several types of cancer such as hepatocellular carcinoma, breast cancer, prostate cancer, gastric cancer and nasopharyngeal cancer.(30-34) One study showed that miR130a expression is highly expressed in NSCLC tissue, and is associated with lymph node metastasis, staging of the tumour node metastasis and poor prognosis.(35) However, there are also contradictory studies, where another group showed that miR130a-3p was expressed at low level and may serve as a tumour suppressor miRNA in NSCLCs. (17) In lung cancer, it is reported that miR130a expression was also reduced and acts as a tumour suppressor by downregulating TGF- $\alpha$.(36) Previous reports showed that overexpressing miR-130a in A549 and Calu-3 cells subdued the proliferative activity of both cells.(33)

Hence, in this study we hypothesised that knocking down miR130 in A549 cell line may enhance the proliferation and migration of the cancer cells. Consistent to the hypothesis, we found that suppression of miR130$3 p$ and $-5 p$ significantly increased the growth rate of A549 as compared to control. In addition to that, we showed that the suppression of miR130-3p significantly increased the migration potential of A549 cells, suggesting the tumour suppressor roles of miR130a-3p in NSCLC.

Meanwhile, in a previous study, overexpression of miR-130a in A549 lung cancer cells was shown to suppress the migratory activity of the A549 cells by targeting TGFA. (36) Tumour suppressor of miR130a was also observed in breast cancer of where the miR130a was expressed at low levels. The silencing of miR130a-3p significantly increased cellular proliferation, migration and invasion of the breast cancer, while overexpression of miR130a-3p had shown the opposite effects.(13) miR130a has also been shown to function as tumour suppressor in glioma, prostate carcinoma and chronic myeloid leukemia $(33,37,38)$, suggesting it's potential therapeutic target for cancer patients. To predict the target gene of miR130a, computational prediction using target gene predictions, combinatorial analysis of gene ontology (GO), miRanda-miRSVR, KEGG analysis and protein-protein interaction (PPI) network was performed by our group, and it was suggested that miR130a-3p may regulate MET, ACVR1 and BCL11A while POU4F2, NLK,
SPOPL, PPARGC1A, BCL11A and ARID4B are observed as target genes of miR130a-5p.(39) The MET, ACVR1 and BCL11A are all listed in The Catalogue of Somatic Mutations in Cancer (COSMIC) Cancer Gene Census (CGC) as cancer-driving gene.(40) MET is a transmembrane tyrosine kinase that are frequently reported in various types of cancers including in NSCLC and colorectal cancer. $(41,42)$ Study in lung cancer cell line has shown that the MET was involved in the regulation of cell migration and invasion.(17) On the other hand, ACVR1, or Activin receptor 1 , has been identified as one of the target gene that regulate metastasis, angiogenesis and lymph angiogenesis in gastric cancer.(43) The other potential target for miR130a is a proto-oncogene BCL11A, which was shown to enhance stemness and promote progression of breast cancer thorough activation of Wnt/ $\beta$-catenin signaling.(44) Interestingly, this BCL11A is also a potential target of miR130-5p. Besides that, POU4F2, NLK, SPOPL, PPARGC1A, BCL11A and ARID4B are also identified as the potential target genes regulated by miR130-5p. The transcription factor POU4F2 was recently reported to promote colorectal cancer cell migration and invasion through the regulation of hedgehog signaling-mediated epithelial-mesenchymal transition.(45)

The Nemo-like kinase (NLK) was reported earlier to boosts cell proliferation and cell cycle progression in colorectal cancer.(46) On the other hand, the molecular mechanisms of SPOP-like (SPOPL) gene have not been explored in cancer studies at present but it was reported to be a potential valuable molecular marker of medulloblastoma. (47) Similarly, the molecular mechanism of the peroxisome proliferator-activated receptor gamma (PPARGC1A) is not well reported. However, it has shown as a promising biomarker for metastatic progression of lung cancer to brain as supported by xenograft tail vein colonization assays.(48) Similar to NLK, the molecular function of the ARID4B is not clearly described but it has shown to be a potential therapeutic target for prostate cancer.(49) The identified target genes of miR130a are reported to be involved in various cancer. However, further analysis for experimental validation between miR130a-3p, miR130a-5p and its associated target genes are needed to further understand their involvement in the regulation of carcinogenesis in NSCLC.

One of the keys finding in this study is that knockdown of miR130a-3p was sequence specific at the 3' of miR130a gene in A549 cell line, while the expression of miR130a$5 p$ was suppressed when the CRISPR/Cas9 was designed to target at the SL region of the mature miR-130a gene. 
Applied together, the suppression of mature miR130a$3 p$ and $-5 p$ significantly increased cell viability of A549 cells. The finding is consistent with the previous study that observed increased proliferation of NSCLS cell lines upon transfection with the miR130a-5p inhibitor, and the mechanism of the NSCLC progression relies on NF$\kappa \mathrm{B}$ activation as regulated by the miR130a-5p/ RUNX2/ STK32A axis.(50) As for the miR130a-3p, there is no study reported so far using knockdown system in NSCLC model system. Although not very well study in the NSCLC, other have reported that miR130a-3p able to promote cell proliferation and invasion by targeting estrogen receptor $\alpha$ and androgen receptor in cervical cancer.(51) Recent study has also reported the involvement of miR130a-3p in modulating cell proliferation and cell cycle production in ovarian epithelial carcinoma.(52) On the other hand, we have observed in our study that the suppression of mature miR130a-3p significantly increased migratory potential of A549 cells as shown by scratch wound healing assay, attachment and detachment assays. Although there is no study using knockdown system to support this finding, study on hypoxic A549 cells has demonstrated an anti-tumour role of miR130a-3p by affecting the numbers of migration and invasion cells, as well as tumour growth in vivo.(53)

Furthermore, in colorectal cancer, miR130a-3p has been shown to regulate cell proliferation and invasion, and able to induce apoptosis through direct interaction with the colon cancer associated transcript 1 (CCAT1).(54) Taken together, all these studies have revealed the roles of miR130a in mediating the carcinogenesis suggesting it's potential target for the development of biomarker or targeted therapy. With this encouraging preliminary data, the next step would be to further investigate the suppression of miR130a-3p at both pre-clinical and clinical level to study the safety, specificity and efficiency of the system in NSCLC targeted therapy.

\section{Conclusion}

In conclusion, we have succeeded in suppressing the expression of miR130a-3p and -5p in the A549 NSCLC cell line. Our results also highlight that among the three regions in miR-130a, suppression at the $3 p$ region significantly affected both the proliferative activity and migratory ability of the A549 NSCLC. These suggest that miR130a-3p may play a critical role in development and metastasis of lung cancer and could be an excellent therapeutic target for the management of NSCLC.

\section{Acknowledgements}

This work was funded by grants from Short Term Grant, Universiti Sains Malaysia (304.CIPPT.6313203) and Fundamental Research Grant Scheme, Ministry of Education Malaysia (203.CIPPT.6711505). We thank our colleagues from Oncology Laboratory and Regenerative Laboratory, Advanced Medical and Dental Institute (IPPT) who provided insight and expertise that greatly assisted the research.

\section{Authors Contribution}

NAA, NIMS and SRAR were involved in concepting and planning of the research, as well as in the designing of the figures. NAA and NIMS performed the data acquisition. NAA, NIMS, MKA and SRAR involved in the experimental data analysis and interpretation of the results. All authors contribute to the manuscript preparation, KTD and SRAR took parts in giving critical revision of the manuscript.

\section{References}

1. Bray F, Ferlay J, Soerjomataram I, Siegel RL, Torre LA, Jemal A. Global cancer statistics 2018: GLOBOCAN estimates of incidence and mortality worldwide for 36 cancers in 185 countries. CA Cancer. 2018; 68: 394-424.

2. Ohno Y, Koyama H, Yoshikawa T, Matsumoto S, Sugimura K. Lung cancer assessment using MR imaging. Magn Reson Imaging Clin N Am. 2015; 23: 231-44.

3. Wei D, Peng JJ, Gao H, Zhang T, Tan Y, Hu YH. ALDH1 expression and the prognosis of lung cancer: a systematic review and metaanalysis. Heart Lung Circ. 2015; 24: 780-8.

4. Hirsch FR, Scagliotti GV, Mulshine JL, Kwon R, Curran WJ, Wu YL, et al. Lung cancer: current therapies and new targeted treatments. Lancet. 2017; 389: 299-311.

5. He L, Hannon GJ. MicroRNAs: small RNAs with a big role in gene regulation. Nat Rev Genet. 2004; 5: 522-31.

6. Chakraborty C, Sharma AR, Sharma G, Sarkar BK, Lee SS. The novel strategies for next-generation cancer treatment: miRNA combined with chemotherapeutic agents for the treatment of cancer. Oncotarget. 2018; 9: 10164-74.

7. Zhang L, Yang X, Lv Y, Xin X, Qin C, Han X, et al. Cytosolic codelivery of miRNA-34a and docetaxel with core-shell nanocarriers via caveolae-mediated pathway for the treatment of metastatic breast cancer. Sci Rep. 2017; 7: 46186. doi: 10.1038/srep46186.

8. Suresh S, McCallum L, Lu W, Lazar N, Perbal B, Irvine AE. MicroRNAs $130 \mathrm{a} / \mathrm{b}$ are regulated by BCR-ABL and downregulate expression of CCN3 in CML. J Cell Commun Signal. 2011; 5: 18391.

9. Zhou P, Jiang W, Wu L, Chang R, Wu K, Wang Z. miR-301a is a 
candidate oncogene that targets the homeobox gene Gax in human hepatocellular carcinoma. Dig Dis Sci. 2012; 57: 1171-80.

10. Liu L, Nie J, Chen L, Dong G, Du X, Wu X, Tang Y, Han W. The oncogenic role of microRNA-130a/301a/454 in human colorectal cancer via targeting Smad4 expression. PLoS One. 2013; 8: 55532. doi: 10.1371/journal.pone.0055532.

11. Ramalho-Carvalho J, Martins JB, Cekaite L, Sveen A, Torres-Ferreira $\mathrm{J}$, Graça I, et al. Epigenetic disruption of miR-130a promotes prostate cancer by targeting SEC23B and DEPDC1. Cancer Lett. 2017; 385: 150-9.

12. Chen X, Zhao M, Huang J, Li Y, Wang S, Harrington CA, et al. microRNA-130a suppresses breast cancer cell migration and invasion by targeting FOSL1 and upregulating ZO-1. J Cell Biochem. 2018; 119: 4945-56.

13. Kong X, Zhang J, Li J, Shao J, Fang L. MiR-130a-3p inhibits migration and invasion by regulating RAB5B in human breast cancer stem cell-like cells. Biochem Biophys Res Commun. 2018; 501: 486-93.

14. Gao W, Yu Y, Cao H, Shen H, Li X, Pan S, et al. Deregulated expression of miR-21, miR-143 and miR-181a in non small cell lung cancer is related to clinicopathologic characteristics or patient prognosis. Biomed Pharmacother. 2010; 64: 399-408.

15. Sorrentino A, Liu CG, Addario A, Peschle C, Scambia G, Ferlini C. Role of microRNAs in drug-resistant ovarian cancer cells. Gynecol Oncol. 2008; 111: 478-86.

16. Hummel R, Hussey DJ, Haier J. MicroRNAs: predictors and modifiers of chemo- and radiotherapy in different tumour types. Eur J Cancer. 2010; 46: 298-311.

17. Acunzo M, Visone R, Romano G, Veronese A, Lovat F, Palmieri D, et al. miR-130a targets MET and induces TRAIL-sensitivity in NSCLC by downregulating miR-221\&222. Oncogene. 2012; 31: $634-42$.

18. Wei MC, Wang YM, Wang DW. miR-130a-Mediated KLF3 can inhibit the growth of lung cancer cells. Cancer Manag Res. 2021; 13: 2995-3004.

19. Ran FA, Hsu PD, Wright J, Agarwala V, Scott DA, Zhang F. Genome engineering using the CRISPR-Cas9 system. Nat Protoc. 2013; 8: 2281-308.

20. Mali P, Yang L, Esvelt KM, Aach J, Guell M, DiCarlo JE, Norville JE, Church GM. RNA-guided human genome engineering via Cas9. Science. 2013; 339: 823-6.

21. Sanjana NE, Shalem O, Zhang F. Improved vectors and genome-wide libraries for CRISPR screening. Nat Methods. 2014; 11: 783-4.

22. Geer LY, Marchler-Bauer A, Geer RC, Han L, He J, He S, et al. The NCBI BioSystems database. Nucleic Acids Res. 2010; 38: D492-6.

23. Gee HE, Buffa FM, Camps C, Ramachandran A, Leek R, Taylor M, et al. The small-nucleolar RNAs commonly used for microRNA normalisation correlate with tumour pathology and prognosis. Br J Cancer. 2009; 104: 1168-77.

24. Goetsch KP, Niesler CU. Optimization of the scratch assay for in vitro skeletal muscle wound healing analysis. Anal Biochem. 2011; 411: 158-60.

25. Liu Y, Li Y, Wang R, Qin S, Liu J, Su F, et al. MiR-130a-3p regulates cell migration and invasion via inhibition of Smad4 in gemcitabine resistant hepatoma cells. Journal of Experimental \& Clinical Cancer Research, 2019, 35: 19. doi: 10.1186/s13046-016-0296-0.

26. Stark A, Brennecke J, Bushati N, Russell RB, Cohen SM. Animal MicroRNAs confer robustness to gene expression and have a significant impact on 3' UTR evolution. Cell. 2005; 123: 1133-46.

27. Newman MA, Thomson JM, Hammond SM. Lin-28 interaction with the Let-7 precursor loop mediates regulated microRNA processing. RNA. 2008; 14: 1539-49.
28. Piskounova E, Viswanathan SR, Janas M, LaPierre RJ, Daley GQ, Sliz P, et al. Determinants of microRNA processing inhibition by the developmentally regulated RNA-binding protein Lin28. Int $\mathrm{J}$ Biol Chem. 2008; 283: 21310-4.

29. Rybak A, Fuchs H, Smirnova L, Brandt C, Pohl EE, Nitsch R, et $a l$. A feedback loop comprising lin-28 and let-7 controls pre-let-7 maturation during neural stem-cell commitment. Nat Cell Biol. 2008; 10: 987-93.

30. Lund E, Güttinger S, Calado A, Dahlberg JE, Kutay U. Nuclear export of microRNA precursors. Science. 2004; 303: 95-8.

31. Zeng Y, Cullen BR. Structural requirements for pre-microRNA binding and nuclear export by Exportin 5. Nucleic Acids Res. 2004; 32: 4776-85.

32. Boll K, Reiche K, Kasack K, Mörbt N, Kretzschmar AK, Tomm JM, et al. miR-203 and miR-205 jointly repress key oncogenic pathways and are downregulated in prostate carcinoma. Oncogene. 2013; 32: 277-85.

33. Cao Z, Cheng W, Lou T, Zhang N, Pan J, Yin Q, et al. Inhibition of miR-130a expression promotes metastasis of lung squamous carcinoma cells. Int J Clin Exp. 2016; 9: 1612-20.

34. Wang T, Wei J, Xu L, Jia R, Guo C. MiR-130a regulates the proliferation and metastasis of HCC cells through targeting ZEB1/2. Int J Clin Exp Pathol. 2017; 10: 2744-53.

35. Wang XC, Du LQ, Zhang H, Wang YY, Wu HY, Li DG, et al. Expression of miRNA-130a in nonsmall cell lung cancer. Am J Med Sci. 2010; 340: 385-8.

36. Zhang W, Ding Q, Zhang N. MiR-130a inhibits cell proliferation and migration via targeting TGFA in non-small cell lung cancer cells. Int J Clin Exp Pathol. 2017; 10: 117-27.

37. Tang C, Yang Z, Chen D, Xie Q, Peng T, Wu J, et al. Downregulation of miR-130a promotes cell growth and epithelial to mesenchymal transition by activating HMGB2 in glioma. Int J Biochem Cell Biol. 2017; 93: 25-31

38. Zhu X, Lin Z, Du J, Zhou X, Yang L, Liu G. Studies on microRNAs that are correlated with the cancer stem cells in chronic myeloid leukemia. Mol Cell Biochem. 2014; 390: 75-84.

39. Abdollah NA, Zulkifle N, Razak SRA. Computational prediction of miR130a target genes in cancer. Malaysian J Med Health Sci. 2020; 16(Suppl 2): 51-9.

40. Sondka Z, Bamford S, Cole CG, Ward SA, Dunham I, Forbes SA The COSMIC cancer gene census: describing genetic dysfunction across all human cancers. Nat Rev Cancer. 2018; 18: 696-705.

41. Khaled WT, Lee SC, Stingl J, Chen X, Ali HR, Rueda OM, et al. BCL11A is a triple-negative breast cancer gene with critical functions in stem and progenitor cells. Nat Commun. 2015; 6: 5987. doi: $10.1038 /$ ncomms6987.

42. Wang S, Qiu J, Liu L, Su C, Qi L, Huang C, et al. CREB5 promotes invasiveness and metastasis in colorectal cancer by directly activating MET. J Exp Clin Cancer Res. 2020; 39: 168. doi: 10.1186/s13046-020-01673-0.

43. Sun Z, Liu C, Jiang WG, Ye L. Deregulated bone morphogenetic proteins and their receptors are associated with disease progression of gastric cancer. Comput Struct Biotechnol J. 2020; 18: 177-88.

44. Zhu L, Pan R, Zhou D, Ye G, Tan W. BCL11A enhances stemness and promotes progression by activating $\mathrm{Wnt} / \beta$-catenin signaling in breast cancer. Cancer Manag Res. 2019; 11: 2997-3007.

45. Guo K, Wang P, Zhang L, Zhou Y, Dai X, Yan Y, et al. Transcription factor POU4F2 promotes colorectal cancer cell migration and invasion through hedgehog-mediated epithelial-mesenchymal transition. Cancer Sci. 2021; 112: 4176-86.

46. Li SZ, Zhen F, Li J, Shu QP, Zhang HH, Xu J, et al. Nemo-like kinase (NLK) primes colorectal cancer progression by releasing the E2F1 
complex from HDAC1. Cancer Lett. 2018; 431: 43-53.

47. Hu Y, Yang L, Zhang M, Huang Z, Lin J, Zhang N. Expression and clinical relevance of SPOPL in medulloblastoma. Oncol Lett. 2017; 14: 3051-6.

48. Li JD, Feng QC, Qi Y, Cui G, Zhao S. PPARGC1A is upregulated and facilitates lung cancer metastasis. Exp Cell Res. 2017; 359: 356-60.

49. Wu RC, Young IC, Chen YF, Chuang ST, Toubaji A, Wu MY. Identification of the PTEN-ARID4B-PI3K pathway reveals the dependency on ARID4B by PTEN-deficient prostate cancer. Nat Commun. 2019; 10: 4332. doi: 10.1038/s41467-019-12184-8.

50. Ma F, Xie Y, Lei Y, Kuanh Z, Liu X. The microRNA-130a-5p/ RUNX2/STK32A network modulates tumor invasive and metastatic potential in non-small cell lung cancer. BMC Cancer. 2020; 20: 580. doi: 10.1186/s12885-020-07056-0.

51. Fan Q, Huang T, Sun X, Yang X, Wang J, Liu Y, et al. miR-130a-3p promotes cell proliferation and invasion by targeting estrogen receptor $\alpha$ and androgen receptor in cervical cancer. Exp Ther Med. 2021; 21: 414. doi: 10.3892/etm.2021.9858.

52. Alrashed MM, Ahmad M, Yongmei W, Xiuyeng W. Differential expression of miR-130a-3p modulate ovarian epithelial carcinoma (OEC) cell development and could be a biomarker for OEC. J Reprod Immunol. 2021; 145: 103310. doi: 10.1016/j.jri.2021.103310.

53. Shi J, Wang H, Feng W, Huang S, An J, Qui Y, Wu K. MicroRNA130a targeting hypoxia-inducible factor 1 alpha suppresses cell metastasis and Warburg effect of NSCLC cells under hypoxia. Life Sci. 2020; 255: 117826. doi: 10.1016/j.lfs.2020.117826.

54. Jing J, Zhao X, Wang J, Li T. Potential diagnostic and prognostic value and regulatory relationship of long noncoding RNA CCAT1 and miR-130a-3p in clear cell renal cell carcinoma. Cancer Cell Int. 2021; 21: 68. doi: 10.1186/s12935-021-01757-7. 